\title{
UTILIZATION OF INTEGRATED PROCESS CONTROL, DATA CAPTURE, AND DATA ANALYSIS IN CONSTRUCTION OF ACCELERATOR SYSTEMS *
}

\author{
V. Bookwalter, B. Madre, J. P. Ozelis, C. Reece ${ }^{+}$ \\ Thomas Jefferson National Accelerator Facility, Newport News, VA. 23606, USA
}

\begin{abstract}
Jefferson Lab has developed a web-based system that integrates commercial database, data analysis, document archiving and retrieval, and user interface software into a coherent knowledge management product called Pansophy. Pansophy provides key tools for the successful pursuit of major projects such as accelerator system development and construction by offering elements of process and procedure control, data capture and review, and data mining and analysis. Today, Pansophy is used in Jefferson Lab's SNS superconducting linac construction effort as a means for structuring and implementing the QA program, for process control and tracking, and for cryomodule test data capture and presentation/analysis. Development of Pansophy continues, with improvements to data queries and analysis functions that are the cornerstone of its utility. In this paper the present configuration and operational environment of Pansophy is described, along with future development goals. Additionally, specific examples of its use in an accelerator construction project will be presented.
\end{abstract}

\section{MOTIVATION AND APPROACH}

Pansophy, a web-based system conceived as a vehicle for collecting, managing and exploiting the data from SRF accelerator subsystem development and production efforts, began development in early 2000 [1]. Because data from the construction of the original CEBAF accelerator was saved in paper form, it was cumbersome to search, and trends were difficult to spot. Some electronic files, often in the form of Excel spreadsheets, existed on individual computers, but there was no coherent effort to save data in a searchable electronic format. This resulted in hesitancy to search the archived documentation when data were needed. It was believed that if data could be made immediately available, and trends observed in near real-time, a feed-forward mechanism could then be exploited, and lessons learned could be applied quickly.

To make the most efficient use of development resources, the system was conceived as a custom integration of several commercial software packages.

Since its initial development, Pansophy has become a vital resource for documenting and processing data from various SRF Institute accelerator projects. Staff members within the SRF Institute and Accelerator Division have begun making extensive use of its capabilities.

\section{GENERAL DESCRIPTION}

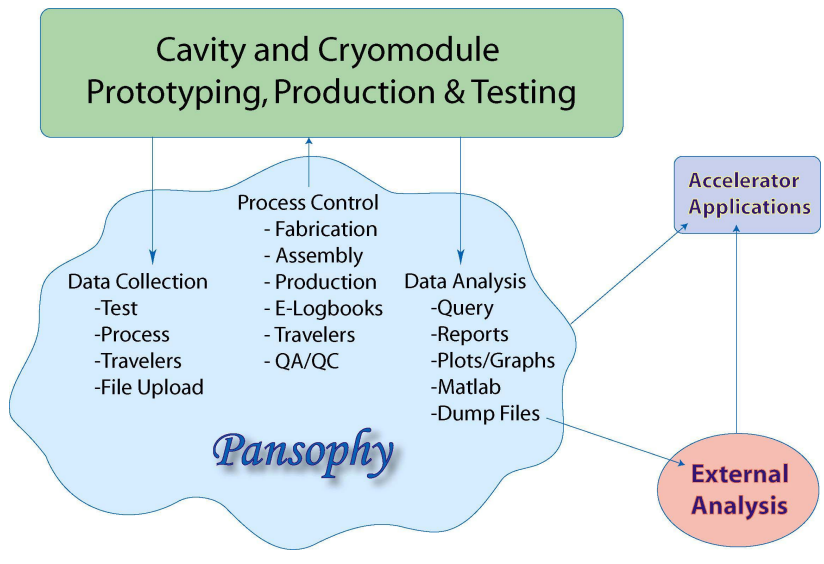

Figure 1: Overview of Pansophy

\section{Travelers}

The traveler, a central component of Pansophy, defines and controls a process while gathering data particular to a part or assembly. It also defines a unit of the database structure. Travelers are initially generated by a scientific or technical staff member using a Microsoft Word ${ }^{\circledR}$ template, while Adobe Acrobat $\AA$ provides the final format for archival of an electronic version of approved travelers. DocuShare ${ }^{\circledR}$, a document management software tool from Xerox, manages, archives, and provides controlled access to travelers. The user interface is written using Macromedia's ColdFusion ${ }^{\circledR}$, and an automated process converts the document from the MS-Word ${ }^{\circledR}$ format to ColdFusion Markup Language (CFML). There are currently over 100 different types of travelers, which have collectively been used about 2000 times. Traveler data, entered online via a web browser, are directly uploaded into an Ingres ${ }^{\circledR}$ database.

\section{Traveler Features}

In addition to the process control and data capture functions described above, travelers also incorporate "hold points," a feature that prohibits the completion of a traveler or section of a traveler (and therefore process) until an authorized supervisor reviews the current data. Additionally, the data recorded by the travelers are frozen once the traveler is closed. These features contribute to satisfying quality assurance requirements.

A specialized Non-Conformance Report (NCR) was developed to track situations where inspections, processes, or tests have uncovered deviations from acceptable

*Work supported by the U. S. Department of Energy contract DE-AC0584ER40150

+bookwalt, madre, ozelis, reece@jlab.org 
parameters. A link is provided on each traveler to initiate an NCR, which is automatically emailed to the responsible engineer for resolution. Upon resolution and close of an NCR, the results are automatically emailed to the NCR initiator and the resident inventory manager. This automated process ensures the appropriate project feedback is achieved [2].

Occasionally activities such as Electron Beam Welding or Niobium Inventory Tracking may require a custom traveler design. This can be easily accommodated.

\section{Access Control and Administration}

The SRF Institute at Jefferson Lab has developed a hierarchical, (WBS) project-based traveler naming convention consisting of five components, plus a revision number and a sequence number. Authorized users can use administrative pages to add new traveler components, modify the header information in a traveler, and view current information.

Pansophy is divided into functional areas and tasks are divided into levels. Authorized users are assigned privilege levels, thus providing secured access to the various functional areas. For instance, supervisors can have additional security levels for traveler data approval, while administrators have appropriate security levels for Pansophy configurations only. Most Pansophy security and administration functions can be performed online.

\section{Logbooks}

Pansophy's electronic Logbooks provide a mechanism for recording time-stamped, non-editable, easily searchable entries of experimental setups, procedures, data, and other information. In addition to this customary usage, these e-logs have proven valuable as a source of information for traveler development.

\section{QC/QA functions}

As Pansophy usage increased, additional functionality was added to facilitate meeting QC/QA program requirements. A calibration management tool was added which permits users to enter devices into a database with required inspection dates and durations and calibrator information. The tool automatically emails calibration reminders to device owners, as well as allowing owners to track the location of devices and instrumentation.

In addition to the calibration system, a maintenance utility based on similar requirements has been added. It is presently used to track and record maintenance status of vacuum equipment operating in both the accelerator facility (CEBAF) and the SRF Institute.

\section{USE IN ACCELERATOR PRODUCTION}

Recent Pansophy development has focused on providing and optimizing database query functionality.
This functionality provides the essential capability for data mining of the traveler system, a primary motivation for Pansophy. Both pre-defined and user-defined queries have been implemented.

Pre-defined queries include simple searches based on Cavity ID, Serial Number, and Vendor ID. Once user input has been entered, all travelers whose data fields contain this label are searched for matches to the user input. A list of travelers is presented to the user with links to the traveler data, allowing the user to quickly find a traveler for a particular cavity and then directly access that cavity's data.

Other pre-defined queries include searches of NCR status (open/closed/all), listings of all instantiations of a selected traveler, and tabular listings of all data fields for all instantiations of a traveler. The NCR search provides an efficient means of tracking NCR status. The data field grids provide overviews of traveler-generated data and allow for initial review of current results from a given production process, which enables feedback to production managers and engineers.

The user-defined query is one of the most powerful, useful features of the Pansophy system. Initially, users are presented with a list of travelers from which to select. When a particular traveler is selected, a listing of available data fields for that traveler is displayed, grouped by traveler page number (Figure 2). The user then selects the data of interest, defines the Boolean operators to be used $(>,<,=$, LIKE, etc.), and selects the operand for the query (Figure 3 ). The user submits the query to the database interactively and is presented with a spreadsheet-style listing of the requested data. This data can then be transferred to an application such as Excel for further analysis (see Fig. 4). Queries and analyses like those in Figure 4 provide a quick and efficient means for tracking trends and performance during the production run.

\section{FUTURE DEVELOPMENT}

The ultimate functionality and utility of Pansophy is explicitly linked to the ability by users to extract, analyze, and benefit from the data captured. Recognizing this, future development of Pansophy will concentrate on developing even more flexible and useful query mechanisms and data output options.

Additionally, the integration of analysis tools such as Matlab ${ }^{\circledR}$ will be pursued. This will enable users to develop semi-custom stored query /analysis suites, so that data can be routinely analyzed in a consistent manner.

In addition to analysis of data resulting from operation of query logic, high-level "views" are planned, where one can chose a component (cryomodule, for example), and then access performance and fabrication data for that component, and some fraction of its subcomponents, using a graphical interface. This interface would allow one to "drill down" to the sub-component level and generate static reports of relevant parameters. Such a feature is expected to be extremely useful in the commissioning phase of installed accelerator components. 


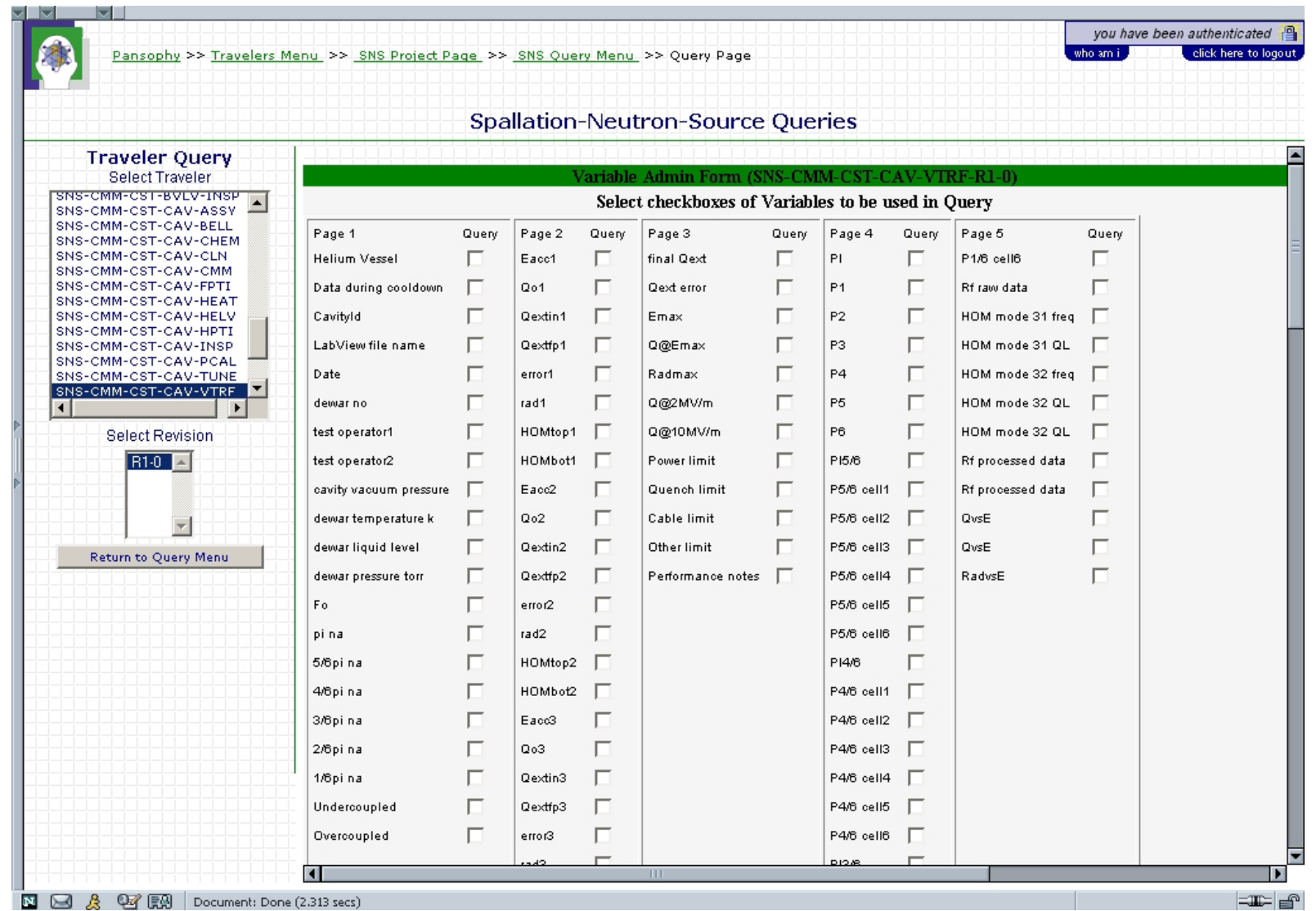

Figure 2. Traveler data field listing used for generating user-defined query.

\begin{tabular}{|c|c|c|c|c|}
\hline \multicolumn{5}{|c|}{ Spallation-Neutron-Source Traveler Queries } \\
\hline paze_var & |abeel & prattpe & Operator & Operand \\
\hline plchkbx1 & Helium Vessel & checkbox & $\overline{A L L} \nabla$ & $\nabla$ \\
\hline pltext1 & CavityId & varchar & $\mathrm{ALL} \quad \nabla$ & \\
\hline pltext3 & Date & date & ALL $\nabla$ & \\
\hline p1 text8 & dewar temperature $\mathrm{k}$ & float & ALL $=$ & \\
\hline p2text2 & Qo1 & float & ALL $\nabla$ & \\
\hline p2text3 & Qextin1 & float & $\mid$ & \\
\hline p2text4 & Qextfp1 & float & $\overline{A L L}=$ & \\
\hline p3text1 & final Qext & float & ALL $\nabla$ & \\
\hline p3text3 & Emax & float & ALL $\nabla$ & \\
\hline p3text4 & $\mathrm{Q} @ \operatorname{Emax}$ & float & $\mid$ & \\
\hline p3text5 & Radmax & float & $\overline{A L L L}$ & \\
\hline p4text8 & PISI6 & float & ALL $\nabla$ & \\
\hline p4text15 & PI4/6 & float & ALL $\nabla$ & \\
\hline p4text22 & PI3/6 & float & ALL $\nabla$ & \\
\hline p4text29 & PI2/6 & float & $\overline{A L L}$ & \\
\hline p4text36 & PI1/6 & float & $\overline{A L L}$ & \\
\hline p5file3 & QvsE & file & ALL $\nabla$ & \\
\hline p5file4s & QveE & file & ALL $\nabla$ & \\
\hline p5file4 & RadvsE & file & $\widehat{A L L} \nabla$ & \\
\hline Submit & & & & \\
\hline
\end{tabular}

Figure 3. Selection of operators and operands for userdefined query
SNS Medium $\beta$ Cavity Field Flatness

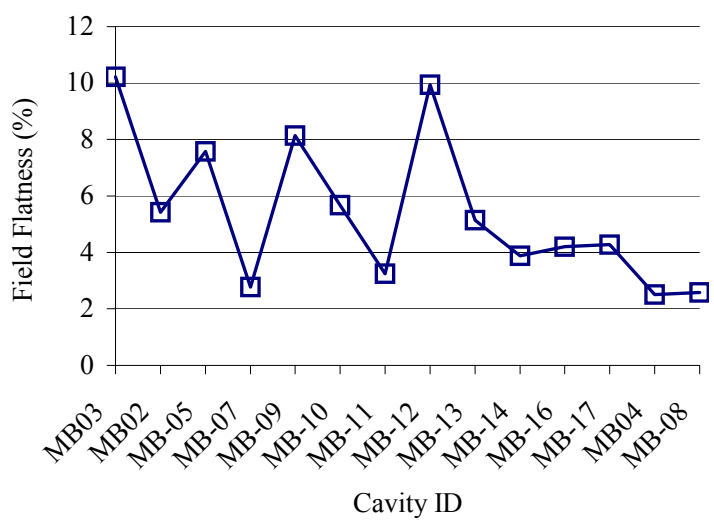

Figure 4. Results of a user-defined query of SNS cavity field flatness $\%$, plotted by cavity.

\section{REFERENCES}

[1] C. Reece et al., "A System for Managing Critical Knowledge for Accelerator Subsystems : Pansophy", PAC2001, Chicago, p. 1189

[2] J. P. Ozelis, "The Jefferson Lab Quality Assurance Program for the SNS Superconducting Linac Accelerator Project", paper TPAG028, PAC2003. 